\title{
WEIGHTED MAXIMUM OVER MINIMUM MODULUS OF POLYNOMIALS, APPLIED TO RAY SEQUENCES OF PADÉ APPROXIMANTS
}

\author{
D.S. LUBINSKY
}

\begin{abstract}
Let $a \geq 0, \varepsilon>0$. We use potential theory to obtain a sharp lower bound for the linear Lebesgue measure of the set

$$
\left\{r \in[0,1]: r^{a n} \frac{\max _{|t|=1}|P(t)|}{\min _{|t|=r}|P(t)|} \leq \varepsilon^{n}\right\} .
$$

Here $P$ is an arbitrary polynomial of degree $\leq n$. We then apply this to diagonal and ray Padé sequences for functions analytic (or meromorphic) in the unit ball. For example, we show that the diagonal $\{[n / n]\}_{n=1}^{\infty}$ sequence provides good approximation on almost $\frac{1}{8}$ of the circles centre 0 , and the $\{[2 n / n]\}_{n=1}^{\infty}$ sequence on almost $\frac{1}{4}$ of such circles.
\end{abstract}

\section{INTRODUCTION}

Let $f$ be a function analytic at 0 , and hence possessing a Maclaurin series there. Recall that if $m, n \geq 0$, the $(m, n)$ Padé approximant to $f$ is a rational function

$$
[m / n](z)=(p / q)(z)
$$

where $p, q$ are polynomials of degree $\leq m, n$ respectively, with $q$ not identically zero, and

$$
(f q-p)(z)=O\left(z^{m+n+1}\right) .
$$

The order relation indicates that the coefficients of $1, z, z^{2}, \ldots z^{m+n}$ in the Maclaurin series of the left-hand side vanish. For an introduction to the subject, see [1].

The convergence theory of Padé approximation is rich and complex. It is known that if $f$ is analytic at 0 , and meromorphic in the whole plane, then $\{[n / n]\}_{n=1}^{\infty}$ converges in measure, and in capacity - the Nuttall-Pommerenke Theorem [13], [14]. More generally, given sequences of positive integers $\left\{m_{k}\right\}_{k=1}^{\infty},\left\{n_{k}\right\}_{k=1}^{\infty}$ that tend to $\infty$ in such a way that for some fixed $\lambda \geq 1$,

$$
\frac{1}{\lambda} \leq \frac{m_{k}}{n_{k}} \leq \lambda, k \geq 1
$$

and given $r, \varepsilon>0$,

$$
m_{2}\left\{z:|z| \leq r \text { and }\left|f-\left[m_{k} / n_{k}\right]\right|(z)>\varepsilon^{n_{k}}\right\} \rightarrow 0, k \rightarrow \infty .
$$

Here $m_{2}$ denotes planar measure, and it may be replaced by capacity. There are deeper analogues for functions with branchpoints [20], [21].

One unfortunate feature of the theorem is that it really requires $f$ to be meromorphic in $\mathbb{C}$. There are functions analytic in the unit ball for which $\{[n / n]\}_{n=1}^{\infty}$ does

Date: May 18, 2001

1991 Mathematics Subject Classification: 30E10, 30C15, 31A15, 41A21. 
not converge in capacity or measure in any ball, no matter how small, contained in the unit ball [6], [7], [15]. Such counterexamples suggest that there is nothing positive that can be said about full diagonal Padé sequences for functions analytic in the unit ball. For subsequences, there is the still unresolved Baker-Gammel-Wills Conjecture, and its cousins - see [1], [8], [9], [22].

The main point of this paper is that nevertheless, there is something positive (and we believe significant) that can be said in this setting. For example, $\{[n / n]\}_{n=1}^{\infty}$ provides good approximation on almost $\frac{1}{8}$ of the circles centre 0 , within the unit ball. This may be viewed as a much sharper form of the results of [10], where it was shown that $[n / n]$ provides approximation on a set of positive proportion, independently of $n$.

The essential ingredient of our development is an inequality for minima and maxima of polynomials. To explain its origin, let us assume for simplicity that $f$ is analytic in $\{z:|z| \leq 1\}$. Then if $[m / n]=p / q$, there is the error formula

$$
\frac{(f q-p)(z)}{z^{m+n+1}}=\frac{1}{2 \pi i} \int_{|t|=1} \frac{(f q)(t)}{t^{m+n+1}} \frac{d t}{t-z},|z|<1 .
$$

(It is a simple consequence of Cauchy's integral formula). Estimation in a standard manner leads to

$$
\max _{|z|=r}|f-[m / n]|(z) \leq\left\{r^{m+n} \frac{\max _{|t|=1}|q(t)|}{\min _{|t|=r}|q(t)|}\right\} \frac{C}{1-r}
$$

for $0<r<1$, where $C$ is independent of $m, n, r$. Since in general one knows little about the zeros of $q$, one wishes to estimate the term in \{\} for an arbitrary polynomial $q$ of degree $\leq n$. For how large a set of $r$ can the term in \{\} be small, and hence for how large a set of $r$ can $[m / n]$ provide good approximation on $|z|=r$ ? Thus we have arrived at the following

\section{PROBLEM}

Let $a \geq 0, \varepsilon>0$, and let $P$ be a polynomial of degree $\leq n$. Estimate below

$$
m_{1}\left(\left\{r \in[0,1]: r^{a n} \frac{\max _{|t|=1}|P(t)|}{\min _{|t|=r}|P(t)|}<\varepsilon^{n}\right\}\right) .
$$

Here $m_{1}$ denotes linear Lebesgue measure.

It turns out that using potential theory for external fields [17], we can obtain a sharp lower bound for the linear Lebesgue measure of this set. Estimates for this set that have been derived in the past have usually been obtained via Cartan's lemma, or classical potential theory, but the factor $r^{a n}$ is typically excluded. The novelty here is the inclusion of the weight $r^{a n}$ in the problem $a b$ initio, leading to sharper estimates.

If one use Bernstein's inequality for the growth of polynomials in the complex plane, one sees that for $P$ of degree $\leq n$ and $r \in[0,1]$,

$$
r^{a n} \frac{\max _{|t|=1}|P(t)|}{\min _{|t|=r}|P(t)|} \leq r^{(a-1) n} \frac{\max _{|t|=r}|P(t)|}{\min _{|t|=r}|P(t)|} .
$$

Thus one could formulate a version of the above problem, that involves a (perhaps more appealing) maximum and minimum modulus over the same circle. Instead of the ordinary potentials used below, this version of the problem leads to Green 
potentials (see [11] for the case $a=0$ and [4] for related results). However, the resulting application to Padé approximation is weaker.

In a certain sense, the problem above is complementary to Zolotarev problems [17, Sn. VIII.3, p. 394ff.]. There one wishes to bound below

$$
r^{a n} \max _{|t|=1}|P(t)| / \min _{|t|=r}|P(t)|,
$$

achievable for some $P$, whereas here, we are trying to see for how large a set of $r$, we can obtain a reasonable upper bound for all $P$. In spirit our results are closer to Remez inequalities [2], and indeed, can be reformulated in Remez form.

We shall present our results on the problem above in Section 2, together with its applications to Padé approximation. The proofs are presented in Sections 3 to 6 .

\section{Statement of Results}

For $a \geq 0$, let us define the external field

$$
Q_{a}(r):=a \log r, r>0 .
$$

Moreover, given $c \in(0,1]$, we define the restricted field

$$
Q_{a, c}(r):=\left\{\begin{array}{l}
\infty, r \in[0, c) \\
Q_{a}(r), r \in[c, 1]
\end{array}\right.
$$

and the associated weight

$$
w_{a, c}(r):=\exp \left(-Q_{a, c}(r)\right)=\left\{\begin{array}{l}
0, r \in[0, c) \\
r^{-a}, r \in[c, 1]
\end{array} .\right.
$$

It follows from the elements of potential theory [17, Thm. I.1.3, p. 27] that there is a unique positive Borel measure $\mu_{a, c}$ of total mass 1 , with support $S\left(\mu_{a, c}\right) \subseteq[c, 1]$ such that

$$
\int \log \frac{1}{|x-t|} d \mu_{a, c}(t)+Q_{a, c}(x) \begin{cases}=F_{a, c}, & \text { q.e. } x \in S\left(\mu_{a, c}\right) ; \\ \geq F_{a, c}, & \text { q.e. } x \in[c, 1] .\end{cases}
$$

Here $F_{a, c}$ is a uniquely determined constant; and q.e. stands for quasi-everywhere, that is except on a set of logarithmic capacity zero. As we shall shortly see, q.e. may be dispensed with. The integral in (2.4) is called the potential associated with the measure $\mu_{a, c}$, and we use the notation

$$
U^{\mu_{a, c}}(x):=\int \log \frac{1}{|x-t|} d \mu_{a, c}(t) .
$$

For an introduction to the sort of potential theory we use, see [5], [12] or [17].

The measure $\mu_{a, c}$ is described in the following proposition:

\section{Proposition 2.1}

Let $a \geq 0, c \in(0,1]$. Let

$$
d:=d(a, c):= \begin{cases}1, & a \leq 1 ; \\ \min \left\{1, c\left(\frac{a}{a-1}\right)^{2}\right\}, & a>1 .\end{cases}
$$

(a) The support of $\mu_{a, c}$ is $[c, d]$;

(b) $\mu_{a, c}$ is absolutely continuous with respect to linear Lebesgue measure; 
(c) For $t \in(c, d)$,

$$
\mu_{a, c}^{\prime}(t)=\frac{1}{\pi \sqrt{(t-c)(d-t)}}\left\{1-a+\frac{a \sqrt{c d}}{t}\right\} .
$$

(d) In particular if $a>1$ and $d<1$, then for $t \in(c, d)$,

$$
\mu_{a, c}^{\prime}(t)=\frac{a-1}{\pi t} \sqrt{\frac{d-t}{t-c}} .
$$

The density in (2.8) is familiar from Lorentz's theory of incomplete polynomials [5], [16], [17], [18]. This is scarcely surprising as the weight $r^{a n}$ was also used there, though in a different setting.

The function

$$
G_{a}(c):=U^{\mu_{a, c}}(-1)-F_{a, c}, c \in(0,1),
$$

is needed in the formulation of our results. We first list some of its main features:

\section{Proposition 2.2}

Fix $a \geq 0$.

(a) $G_{a}$ is a strictly decreasing continuous function of $c \in(0,1)$.

(b) If $a>1, G_{a}$ maps $(0,1)$ onto $\mathbb{R}$.

(c) If $a \leq 1, G_{a}$ maps $(0,1)$ onto $(-\infty,-(1-a) \log (3+\sqrt{8}))$.

(d) $G_{a}$ admits the representation

$$
\begin{aligned}
G_{a}(c)= & -a \log \left(\frac{d+c}{d-c}+\sqrt{\left(\frac{d+c}{d-c}\right)^{2}-1}\right) \\
& -\log \left(\frac{d+c+2}{d-c}+\sqrt{\left(\frac{d+c+2}{d-c}\right)^{2}-1}\right)-a \log \frac{d-c}{4} \\
& -2 a \log \left(1-\left[\frac{d+c}{d-c}-\sqrt{\left(\frac{d+c}{d-c}\right)^{2}-1}\right]\left[\frac{d+c+2}{d-c}-\sqrt{\left(\frac{d+c+2}{d-c}\right)^{2}-1}\right]\right),
\end{aligned}
$$

where $d=d(a, c)$ is given by (2.6).

Since $G_{a}$ is strictly decreasing and continuous, it has an inverse function, which we denote by $G_{a}^{[-1]}$. We define

$$
H_{a}(\varepsilon):=G_{a}^{[-1]}(-\log \varepsilon),
$$

for all $\varepsilon>0$ for which $-\log \varepsilon$ lies in the range of $G_{a}$ : this range is given in (2.12) below. We note that $H_{a}$ is a strictly increasing continuous function. We may now state our main result: 


\section{Theorem 2.3}

Let $a \geq 0$, and let

$$
\begin{array}{ll}
\varepsilon \in(0, \infty), & \text { if } a>1 \\
\varepsilon \in\left((3+\sqrt{8})^{1-a}, \infty\right), & \text { if } a \leq 1 .
\end{array}
$$

(a) If $n \geq 1$ and $P$ is a polynomial of degree $\leq n$, then

$$
m_{1}\left\{r \in[0,1]: r^{a n} \frac{\max _{|t|=1}|P(t)|}{\min _{|t|=r}|P(t)|}<\varepsilon^{n}\right\} \geq H_{a}(\varepsilon) .
$$

(b) This is sharp in the sense that we may find for large enough $n$ a polynomial $P$ for which the left hand side in (2.13) is as close to $H_{a}(\varepsilon)$ as we please.

The restrictions on $\varepsilon$ arise from the fact that the set in (2.13) may be empty if $\varepsilon$ is too small. For example, if $a=1$, and we take $P(t)=t^{n}$, then

$$
r^{a n} \frac{\max _{|t|=1}|P(t)|}{\min _{|t|=r}|P(t)|}=1,
$$

so that set is empty unless $\varepsilon>1$. We shall present a table of values of $H_{a}(1)$ after Theorem 2.4. For the moment, we just note two elegant special cases, $(a=2,3$ and $\varepsilon=1$ ), for which we can evaluate $H_{a}(\varepsilon)$ (see Lemma 3.2):

$$
\begin{aligned}
& m_{1}\left\{r \in[0,1]: r^{2 n} \frac{\max _{|t|=1}|P(t)|}{\min _{|t|=r}|P(t)|}<1\right\} \geq \frac{1}{8} \\
& m_{1}\left\{r \in[0,1]: r^{3 n} \frac{\max _{|t|=1}|P(t)|}{\min _{|t|=r}|P(t)|}<1\right\} \geq \frac{1}{4}
\end{aligned}
$$

We again emphasise that $\frac{1}{4}$ and $\frac{1}{8}$ are sharp.

We now turn to results on Padé approximation. Note that if $\lambda$ is fixed and $m=\lambda n$, then $m+n=(\lambda+1) n$, so the $[\lambda n / n]$ Padé approximant corresponds to $a=\lambda+1$ in (2.13).

\section{Theorem 2.4}

Let $f$ be analytic at 0 , and let $f$ be meromorphic in $\{z:|z|<1\}$. Let $\lambda$ be a positive integer, and

$$
0<\delta<H_{\lambda+1}(1)
$$

Then there exists $\varepsilon=\varepsilon(\delta) \in(0,1)$ and $n_{0}>0$ such that for $n \geq n_{0}$,

$$
m_{1}\left\{r \in[0,1]: \max _{|z|=r}|f-[\lambda n / n]|(z) \leq \varepsilon^{n}\right\} \geq H_{\lambda+1}(1)-\delta .
$$

Thus $[\lambda n / n]$ provides good approximation to $f$ on almost $H_{\lambda+1}(1)$ of the circles centre 0 in the unit ball. Of course the restriction that $\lambda$ be an integer is inessential, and is needed only in ensuring that $\lambda n$ is an integer; one could consider for general $\lambda>0$, ray sequences $\left\{\left[m_{k} / n_{k}\right]\right\}_{k=1}^{\infty}$ with

$$
\lim _{k \rightarrow \infty} m_{k} / n_{k}=\lambda \text {. }
$$


A similar result is true for $\{[n / \lambda n]\}_{n=1}^{\infty}$ : suppose for simplicity that $f(0) \neq 0$. Then $1 / f$ is also analytic at 0 and meromorphic in the unit ball. Since

$$
[n / \lambda n]_{f}=1 /[\lambda n / n]_{1 / f},
$$

where the subscript indicates the function from which the approximant is formed, applying Theorem 2.4 to $1 / f$ yields a matching result for $\left\{[n / \lambda n]_{f}\right\}_{n=1}^{\infty}$.

Following is a table of values of $H_{\lambda+1}(1)$, prepared using Mathematica 3.0:

\begin{tabular}{|l||l||l||}
\hline \hline Sequence & Parameter $a$ & $H_{\lambda+1}(1)=$ Proportion of Good Circles \\
{$[n / n]$} & 2 & $\frac{1}{8}$ \\
{$[2 n / n]$ or $[n / 2 n]$} & 3 & $\frac{1}{4}$ \\
{$[3 n / n]$ or $[n / 3 n]$} & 4 & $34.34 \%$ \\
{$[4 n / n]$ or $[n / 4 n]$} & 5 & $41.46 \%$ \\
{$[5 n / n]$ or $[n / 5 n]$} & 6 & $47.07 \%$ \\
{$[10 n / n]$ or $[n / 10 n]$} & 11 & $61.22 \%$ \\
{$[20 n / n]$ or $[n / 20 n]$} & 21 & $75.95 \%$ \\
\hline
\end{tabular}

One may also present a generalisation of Theorem 2.4 involving a power of $z$ :

\section{Theorem 2.5}

Let $f$ be analytic at 0 , and let $f$ be meromorphic in $\{z:|z|<1\}$. Let $\lambda$ be a positive integer, and

$$
0 \leq \rho \leq \lambda+1
$$

Let $\varepsilon$ lie in the range of $G_{\lambda+1-\rho}$. Then given

$$
0<\delta<H_{\lambda+1-\rho}(\varepsilon),
$$

there exists $n_{0}>0$ such that for $n \geq n_{0}$,

$$
m_{1}\left\{r \in[0,1]: \max _{|z|=r} \frac{|f-[\lambda n / n]|}{|z|^{\rho n}}(z) \leq \varepsilon^{n}\right\} \geq H_{\lambda+1-\rho}(\varepsilon)-\delta .
$$

We shall also present a result involving errors of rational approximation. For $f$ analytic in $\{z:|z| \leq 1\}$, except possibly for poles of total multiplicity $\ell$, let us define for $n \geq \ell$, the error in approximation of $f$ by rational functions of type $(n, n)$, on the unit circle, by

$$
\rho_{n}(f):=\inf \left\{\left\|f-\frac{p}{q}\right\|_{L_{\infty}(|z|=1)}: \operatorname{deg}(p), \operatorname{deg}(q) \leq n ; q \text { not identically } 0\right\} .
$$


Then we have:

\section{Theorem 2.6}

Let $f$ be analytic in $\{z:|z| \leq 1\}$, except for poles of total multiplicity $\ell$, none at 0 . Then given $\varepsilon>1$, and $\delta>0$, there exists $n_{0}$, such that for $n \geq n_{0}$,

$$
m_{1}\left\{r \in[0,1]: \max _{|z|=r} \frac{|f-[n / n]|}{\rho_{n-\ell}(f)}(z) \leq \varepsilon^{n}\right\} \geq H_{1}(\varepsilon)-\delta .
$$

For example, if we choose $\varepsilon=2$, a Mathematica 3.0 calculation shows that

$$
m_{1}\left\{r \in[0,1]: \max _{|z|=r} \frac{|f-[n / n]|}{\rho_{n-\ell}(f)}(z) \leq 2^{n}\right\} \geq 0.0588 \ldots-\delta .
$$

This paper is organised as follows: in Section 3, we prove Propositions 2.1 and 2.2. In Section 4, we prove Theorem 2.3(a), and in Section 5, we prove Theorem 2.3(b). Finally, in Section 6, we prove Theorems 2.4-6.

\section{The Equlibrium Measure and Potential}

We remark that the proof of Proposition in 2.1 has points of contact with several proofs in the theory of incomplete polynomials [5], [16], [18], [19]. Nevertheless, we provide all the details, as we do not believe our result follows in full generality from existing results, and in any event, to patch together results from various sources would be awkward. So we proceed directly from the standard text [17].

\section{Proof of Proposition 2.1}

(a) Now $x Q_{a, c}^{\prime}(x)=a$ is increasing in $[c, 1]$, so $S\left(\mu_{a, c}\right)$ is an interval [17, Thm.IV.1.10, pp.198-9], say,

$$
S\left(\mu_{a, c}\right)=\left[\alpha^{*}, \beta^{*}\right] .
$$

To show that $\alpha^{*}=c$, we use the Mhaskar-Saff $F$-functional

$$
F([\alpha, \beta])=\log \frac{\beta-\alpha}{4}-\frac{1}{\pi} \int_{\alpha}^{\beta} \frac{Q_{a, c}(x)}{\sqrt{(x-\alpha)(\beta-x)}} d x,
$$

for $[\alpha, \beta] \subseteq[c, 1]$. It is known [17, Thm IV.1.5, p.194] that

$$
F\left(\left[\alpha^{*}, \beta^{*}\right]\right)=\max _{[\alpha, \beta] \subseteq[c, 1]} F([\alpha, \beta]),
$$

and only $\left[\alpha^{*}, \beta^{*}\right]$ attains the maximum. Suppose that $\alpha^{*}>c$; we shall derive a contradiction by translating $\left[\alpha^{*}, \beta^{*}\right]$ leftwards to have left endpoint $c$. Let

$$
\delta:=\beta^{*}-\alpha^{*},
$$

and consider $[c, c+\delta] \subset[c, 1]$. Then

$$
\begin{aligned}
F([c, c+\delta]) & =\log \frac{\delta}{4}+\frac{a}{\pi} \int_{c}^{c+\delta} \frac{|\log x|}{\sqrt{(x-c)(c+\delta-x)}} d x \\
& =\log \frac{\beta^{*}-\alpha^{*}}{4}+\frac{a}{\pi} \int_{\alpha^{*}}^{\beta^{*}} \frac{\left|\log \left(t+c-\alpha^{*}\right)\right|}{\sqrt{\left(t-\alpha^{*}\right)\left(\beta^{*}-t\right)}} d t \\
& >\log \frac{\beta^{*}-\alpha^{*}}{4}+\frac{a}{\pi} \int_{\alpha^{*}}^{\beta^{*}} \frac{|\log t|}{\sqrt{\left(t-\alpha^{*}\right)\left(\beta^{*}-t\right)}} d t=F\left(\left[\alpha^{*}, \beta^{*}\right]\right),
\end{aligned}
$$


since $c-\alpha^{*}<0$. This contradiction to (3.2) shows that $\alpha^{*}=c$.

We now maximize over $d$,

$$
F(d):=F([c, d]):=\log \frac{d-c}{4}-\frac{a}{\pi} \int_{0}^{1} \frac{\log (c+t(d-c))}{\sqrt{t(1-t)}} d t .
$$

(We have made a substitution in the integral in (3.1)). Then for $d \in(c, 1)$,

$$
F^{\prime}(d)=\frac{1}{d-c}\left\{1-\frac{a}{\pi} \int_{0}^{1} \frac{t}{\frac{c}{d-c}+t} \frac{d t}{\sqrt{t(1-t)}}\right\} .
$$

Here

$$
\frac{1}{\pi} \int_{0}^{1} \frac{t}{\frac{c}{d-c}+t} \frac{d t}{\sqrt{t(1-t)}}<\frac{1}{\pi} \int_{0}^{1} \frac{d t}{\sqrt{t(1-t)}}=1,
$$

so if $a \leq 1$,

$$
F^{\prime}(d)>\frac{1-a}{d-c} \geq 0, d \in(c, 1) .
$$

Then $F$ is increasing in $(c, 1)$ and

$$
\max _{d \in[c, 1]} F(d)=F(1) .
$$

Hence

$$
S\left(\mu_{a, c}\right)=[c, 1] .
$$

We now turn to the case $a>1$. Here either $F^{\prime}(d)>0, d \in(c, 1)$ in which case again $S\left(\mu_{a, c}\right)=[c, 1]$, or there exists $d \in(c, 1)$ such that $F^{\prime}(d)=0$. In the latter case, such a $d$ satisfies

$$
\frac{a}{\pi} \int_{0}^{1} \frac{t}{\frac{c}{d-c}+t} \frac{d t}{\sqrt{t(1-t)}}=1,
$$

and also $S\left(\mu_{a, c}\right)=[c, d]$. We need the integral

$$
\frac{1}{\pi} \int_{\alpha}^{\beta} \frac{d s}{s \sqrt{(s-\alpha)(\beta-s)}}=\frac{1}{\sqrt{\alpha \beta}}, 0<\alpha<\beta .
$$

One may derive this by contour integral methods, or see [3, no. 3.147.6, p.242]. Setting

we see that

$$
\lambda:=\frac{c}{d-c},
$$

$$
\begin{aligned}
\frac{a}{\pi} \int_{0}^{1} \frac{t}{\frac{c}{d-c}+t} \frac{d t}{\sqrt{t(1-t)}} & =\frac{a}{\pi} \int_{0}^{1}\left(1-\frac{\lambda}{t+\lambda}\right) \frac{d t}{\sqrt{t(1-t)}} \\
& =a\left(1-\frac{\lambda}{\pi} \int_{\lambda}^{\lambda+1} \frac{d s}{s \sqrt{(s-\lambda)(\lambda+1-s)}}\right) \\
& =a\left(1-\sqrt{\frac{\lambda}{\lambda+1}}\right),
\end{aligned}
$$

by (3.4). Then (3.3) becomes

$$
a\left(1-\sqrt{\frac{c}{d}}\right)=1 \Leftrightarrow d=c\left(\frac{a}{a-1}\right)^{2} .
$$


In summary, if $a>1$, we do have the desired formula (2.6).

(b) Since $Q_{a}^{\prime \prime}$ is continuous in [c,1], this follows from [17, Thm. IV.2.2, p.211 ff.].

(c) We use the formula

$$
\mu_{a, c}^{\prime}(x)=\frac{1}{\pi^{2}} \sqrt{\frac{d-x}{x-c}} P V \int_{c}^{d} \frac{(s-c) Q_{a}^{\prime}(s)}{s-x} \frac{d s}{\sqrt{(s-c)(d-s)}}+\frac{D}{\sqrt{(x-c)(d-x)}},
$$

where $P V$ denotes Cauchy principal value, and

$$
D:=\frac{1}{\pi}-\frac{1}{\pi^{2}} \int_{c}^{d} \sqrt{\frac{s-c}{d-s}} Q_{a}^{\prime}(s) d s .
$$

(This follows directly from Thm. IV.3.2 in [17, p.226] by mapping $[c, d]$ onto $[0,1])$. Now using

$$
\frac{s-c}{s(s-x)}=\frac{x-c}{x} \frac{1}{s-x}+\frac{c}{s x}
$$

and the identity $[17$, p. 225 , eqn. (3.20)]

$$
\frac{1}{\pi} P V \int_{c}^{d} \frac{1}{s-x} \frac{d s}{\sqrt{(s-c)(d-s)}}=0, x \in(c, d),
$$

we see that

$$
\begin{aligned}
& \frac{1}{\pi} P V \int_{c}^{d} \frac{(s-c) Q_{a}^{\prime}(s)}{s-x} \frac{d s}{\sqrt{(s-c)(d-s)}} \\
= & 0+\frac{a c}{x} \frac{1}{\pi} \int_{c}^{d} \frac{d s}{s \sqrt{(s-c)(d-s)}} \\
= & \frac{a}{x} \sqrt{\frac{c}{d}},
\end{aligned}
$$

by (3.4). Next,

$$
\begin{aligned}
D & =\frac{1}{\pi}-\frac{a}{\pi^{2}} \int_{c}^{d} \frac{s-c}{s \sqrt{(s-c)(d-s)}} d s \\
& =\frac{1}{\pi}-\frac{a}{\pi}\left(1-\sqrt{\frac{c}{d}}\right),
\end{aligned}
$$

again by (3.4). Of course in the special case $a>1>d$, (3.5) shows that $D=0$. Putting this all together in (3.6) gives

$$
\mu_{a, c}^{\prime}(x)=\frac{1}{\pi} \sqrt{\frac{d-x}{x-c}} \frac{a}{x} \sqrt{\frac{c}{d}}+\frac{1}{\sqrt{(x-c)(d-x)}}\left(\frac{1}{\pi}-\frac{a}{\pi}\left(1-\sqrt{\frac{c}{d}}\right)\right),
$$

which simplifies to (2.7).

(d) In the special case $a>1>d$, we noted that (3.5) gives $D=0$, and then (3.5) and the last equation easily give (2.8).

We remark that it is an easy consequence of (2.7) that $U^{\mu_{a, c}}$ is continuous in $[c, d]$ and hence in $\mathbb{C}$. So the left-hand side of (2.4) is continuous in $x$, and consequently, we can drop the q.e. in the right-hand side of (2.4). 


\section{The Proof of Proposition 2.2}

(a) Note first that

$$
c_{1}<c_{2} \Rightarrow w_{a, c_{1}} \geq w_{a, c_{2}} \text { in }\left[c_{1}, 1\right] .
$$

It then follows that

$$
G_{a}\left(c_{1}\right)=U^{\mu_{a, c_{1}}}(-1)-F_{a, c_{1}} \geq U^{\mu_{a, c_{2}}}(-1)-F_{a, c_{2}}=G_{a}\left(c_{2}\right),
$$

see [17, Cor. I.4.2(a), p.50]. So $G_{a}(c)$ is monotone decreasing. We shall delay the proof that it is strictly decreasing until the end of the proof of (d). To prove that $G_{a}$ is continuous, we note first that $d=d(a, c)$ is continuous in $c$, and then the explicit form (2.7) for $\mu_{a, c}$ implies that $U^{\mu_{a, c}}(-1)$ is continuous in $c$. The continuity of $F_{a, c}$ in $c$ follows similarly from (3.8) below.

(b), (c) Because of the continuity of $G_{a}$, it suffices to compute its left limit at 1 and its right limit at 0 . Let us first compute the former. Recall from (2.9) that

$$
G_{a}(c)=-\int_{c}^{d} \log (1+t) d \mu_{a, c}(t)-F_{a, c} .
$$

Here as $[c, d] \subseteq[0,1]$,

$$
-\log 2 \leq-\int_{c}^{d} \log (1+t) d \mu_{a, c}(t) \leq 0 .
$$

Also, it is known [17, Thm.IV.1.5(b), p.194] that

$$
\begin{aligned}
-F_{a, c} & =F([c, d]) \\
& =\sup _{[\alpha, \beta] \subseteq[c, 1]} F([\alpha, \beta]) \\
& =\sup _{[\alpha, \beta] \subseteq[c, 1]}\left(\log \frac{\beta-\alpha}{4}-\frac{1}{\pi} \int_{\alpha}^{\beta} \frac{Q_{a}(x)}{\sqrt{(x-\alpha)(\beta-x)}} d x\right) .
\end{aligned}
$$

Here as $c \rightarrow 1-$, uniformly for $x \in[c, 1]$

$$
-Q_{a}(x)=a|\log x| \rightarrow 0
$$

while for $[\alpha, \beta] \subseteq[c, 1]$,

$$
\log \frac{\beta-\alpha}{4} \leq \log \frac{1-c}{4} \rightarrow-\infty .
$$

Thus

$$
\lim _{c \rightarrow 1-} G_{a}(c)=\lim _{c \rightarrow 1-}\left(-F_{a, c}+O(1)\right)=-\infty .
$$

Next, we compute $\lim _{c \rightarrow 0+} G_{a}(c)$. We consider two subcases: (1) $a>1$

Here for small enough $c$, and $d=d(a, c)$,

$$
d=c\left(\frac{a}{a-1}\right)^{2}
$$

so uniformly for $x \in[c, d]$,

$$
Q_{a}(x)=a \log c+O(1)
$$


WEIGHTED MAXIMUM OVER MINIMUM MODUlus OF POLYNOMiALS, APPLIED TO RAY SEQUENCES OF PADÉ APPROX

and hence

$$
\begin{aligned}
-F_{a, c} & =F([c, d]) \\
& =\log \left(\frac{d-c}{4}\right)-\frac{1}{\pi} \int_{c}^{d} \frac{Q_{a}(x)}{\sqrt{(x-c)(d-x)}} d x \\
& =\log c+O(1)-a \log c+O(1) \\
& =(a-1)|\log c| \rightarrow \infty, c \rightarrow 0+.
\end{aligned}
$$

Then, together with (3.7), this shows that

$$
\lim _{c \rightarrow 0+} G_{a}(c)=\infty .
$$

(2) $a \leq 1$

Here $d=d(a, c)=1$ for all $c$. A straightforward application of dominated convergence and (2.7) then shows that

$$
\lim _{c \rightarrow 0+} G_{a}(c)=-\frac{1-a}{\pi} \int_{0}^{1} \frac{\log (1+t)}{\sqrt{t(1-t)}} d t+\log \frac{1}{4}-\frac{a}{\pi} \int_{0}^{1} \frac{\log t}{\sqrt{t(1-t)}} d t .
$$

We now use a standard integral from potential theory [17, Example I.3.5, pp.45-46]

$$
\frac{1}{\pi} \int_{-1}^{1} \frac{\log |x-t|}{\sqrt{1-t^{2}}} d t=\log \left|\frac{|x|+\sqrt{|x|^{2}-1}}{2}\right|, x \in \mathbb{R}
$$

where if $x \in[-1,1]$, the right-hand side reduces to $\log \frac{1}{2}$. A linear substitution then yields, for $\alpha<\beta ; y \in \mathbb{R}$,

$$
\begin{aligned}
& \frac{1}{\pi} \int_{\alpha}^{\beta} \frac{\log |s-y|}{\sqrt{(s-\alpha)(\beta-s)}} d s \\
= & \log || \frac{2}{\beta-\alpha}\left(y-\frac{\beta+\alpha}{2}\right)\left|+\sqrt{\left|\frac{2}{\beta-\alpha}\left(y-\frac{\beta+\alpha}{2}\right)\right|^{2}-1}\right|-\log \frac{4}{\beta-\alpha} .
\end{aligned}
$$

Applying this with $[\alpha, \beta]=[0,1]$ and $y=-1$ or 0 in (3.9) gives

$$
\begin{aligned}
\lim _{c \rightarrow 0+} G_{a}(c)= & -(1-a)(\log (3+\sqrt{8})-\log 4) \\
& +\log \frac{1}{4}-a(-\log 4) \\
= & -(1-a) \log (3+\sqrt{8}) .
\end{aligned}
$$

(d) From our representation (2.7) for $\mu_{a, c}^{\prime}$, we see that

$$
\begin{aligned}
G_{a}(c)= & -\frac{1-a}{\pi} \int_{c}^{d} \frac{\log (1+t)}{\sqrt{(t-c)(d-t)}} d t-\frac{a \sqrt{c d}}{\pi} \int_{c}^{d} \frac{\log (1+t)}{t \sqrt{(t-c)(d-t)}} d t \\
& +\log \left(\frac{d-c}{4}\right)-\frac{a}{\pi} \int_{c}^{d} \frac{\log t}{\sqrt{(t-c)(d-t)}} d t .
\end{aligned}
$$


By applying (3.10) with $[\alpha, \beta]=[c, d]$ and $y=-1$ or $y=0$ to this, we see that

$$
\begin{aligned}
G_{a}(c)= & -(1-a)\left\{\log \left(\left[\frac{d+c+2}{d-c}\right]+\sqrt{\left[\frac{d+c+2}{d-c}\right]^{2}-1}\right)-\log \frac{4}{d-c}\right\} \\
& -\frac{a \sqrt{c d}}{\pi} \int_{c}^{d} \frac{\log (1+t)}{t \sqrt{(t-c)(d-t)}} d t+\log \left(\frac{d-c}{4}\right) \\
& -a\left\{\log \left(\left[\frac{d+c}{d-c}\right]+\sqrt{\left[\frac{d+c}{d-c}\right]^{2}-1}\right)-\log \frac{4}{d-c}\right\} .
\end{aligned}
$$

We now use Lemma 3.1 (below) in the integral on the right-hand side of (3.11); we see that then (3.11) simplifies to (2.10), after some straightforward manipulation.

Finally, we use the explicit formula (2.10) to show that $G_{a}$ is a strictly decreasing function of $c$. Firstly, (2.6) shows that $d=d(a, c)$ is analytic in $c$ except if $a \leq 1$ and $c=\left(\frac{a-1}{a}\right)^{2}$ (where the two terms in the minimum in (2.6) are equal). Hence $G_{a}(c)$ is analytic in $c$

(i) for $c \in(0,1)$ if $a \leq 1$;

(ii) for $c \in\left(0,\left(\frac{a-1}{a}\right)^{2}\right)$ and $c \in\left(\left(\frac{a-1}{a}\right)^{2}, 1\right)$ if $a>1$.

Now if there exists $c_{1}<c_{2}$, with

$$
G_{a}\left(c_{1}\right)=G_{a}\left(c_{2}\right),
$$

we have because of monotonicity,

$$
G_{a}^{\prime}(c)=0, c \in\left(c_{1}, c_{2}\right) .
$$

Then analytic continuation and (3.12) shows that respectively

(i) $G_{a}$ is constant in $(0,1)$ if $a \leq 1$;

(ii) $G_{a}$ is constant in either $\left(0,\left(\frac{a-1}{a}\right)^{2}\right)$ or $\left(\left(\frac{a-1}{a}\right)^{2}, 1\right)$ if $a>1$.

We then obtain a contradiction to the results of (b), (c).

We now establish an integral identity, using standard integrals:

\section{Lemma 3.1}

Let $0<\alpha<\beta$. Then

$$
\begin{aligned}
I \quad: \quad=\frac{1}{\pi} \int_{\alpha}^{\beta} \frac{\log (1+x)}{x \sqrt{(x-\alpha)(\beta-x)}} d x \\
\quad=\frac{1}{\sqrt{\alpha \beta}}\left\{\begin{array}{c}
\log \left(1+\frac{\beta+\alpha}{2}\right)+\log \left(\frac{1+\sqrt{1-\left(\frac{\beta-\alpha}{\beta+\alpha+2}\right)^{2}}}{2}\right) \\
+2 \log \left(1-\left[\frac{\beta+\alpha}{\beta-a}-\sqrt{\left(\frac{\beta+\alpha}{\beta-\alpha}\right)^{2}-1}\right]\left[\frac{\beta+\alpha+2}{\beta-a}-\sqrt{\left(\frac{\beta+\alpha+2}{\beta-\alpha}\right)^{2}-1}\right]\right)
\end{array}\right\} .
\end{aligned}
$$

Proof

\section{Step 1: Reduce to standard integrals}


WEIGHTED MAXIMUM OVER MINIMUM MODUluS OF POLYNOMIALS, APPLIED TO RAY SEQUENCES OF PADÉ APPROX

We first map $[\alpha, \beta]$ linearly to $[-1,1]$ and then make the trigonometric substitution $x=\cos \theta$ to get rid of the square root factor, and hence deduce that

$$
\begin{aligned}
I & =\frac{1}{\pi} \int_{0}^{\pi} \frac{\log \left(1+\frac{\beta+\alpha}{2}+\frac{\beta-\alpha}{2} \cos \theta\right)}{\frac{\beta+\alpha}{2}+\frac{\beta-\alpha}{2} \cos \theta} d \theta \\
& =\frac{2}{\beta+\alpha} \log \left(1+\frac{\beta+\alpha}{2}\right) I_{1}+\frac{2}{\beta+\alpha} I_{2},
\end{aligned}
$$

where

$$
\begin{gathered}
I_{1}=\frac{1}{\pi} \int_{0}^{\pi} \frac{d \theta}{1+r \cos \theta} ; \\
I_{2}=\frac{1}{\pi} \int_{0}^{\pi} \frac{\log (1+s \cos \theta)}{1+r \cos \theta} d \theta ;
\end{gathered}
$$

and

$$
r:=\frac{\beta-\alpha}{\beta+\alpha} ; s:=\frac{\beta-\alpha}{\beta+\alpha+2} .
$$

\section{Step 2: Apply standard integrals}

Now $[3$, no. 3.613 .1$, p.366],

$$
I_{1}=\frac{1}{\sqrt{1-r^{2}}}=\frac{\beta+\alpha}{2 \sqrt{\alpha \beta}}
$$

Next, for $|a| \leq 1,|b|<1,[3$, no. 4.397.16, p.594]

$$
I_{3}:=\frac{1}{\pi} \int_{0}^{\pi} \frac{\log \left(1-2 a \cos \theta+a^{2}\right)}{1-2 b \cos \theta+b^{2}} d \theta=\frac{2 \log (1-a b)}{1-b^{2}} .
$$

To evaluate $I_{2}$, we write

$$
\begin{gathered}
s=\frac{-2 a}{1+a^{2}} ; r=\frac{-2 b}{1+b^{2}} \\
\Leftrightarrow a=\frac{-1+\sqrt{1-s^{2}}}{s} ; b=\frac{-1+\sqrt{1-r^{2}}}{r} .
\end{gathered}
$$

Then

$$
\begin{aligned}
I_{2}= & \frac{1}{\pi} \int_{0}^{\pi} \frac{\log \left(1-\frac{2 a}{1+a^{2}} \cos \theta\right)}{1-\frac{2 b}{1+b^{2}} \cos \theta} d \theta \\
= & \left(\log \frac{1}{1+a^{2}}\right) \frac{1}{\pi} \int_{0}^{\pi} \frac{d \theta}{1+r \cos \theta} \\
& +\left(1+b^{2}\right) \frac{1}{\pi} \int_{0}^{\pi} \frac{\log \left(1+a^{2}-2 a \cos \theta\right)}{1+b^{2}-2 b \cos \theta} d \theta \\
= & \left(\log \frac{1}{1+a^{2}}\right) \frac{1}{\sqrt{1-r^{2}}}+\left(1+b^{2}\right) \frac{2 \log (1-a b)}{1-b^{2}},
\end{aligned}
$$

by (3.18) and (3.19). A calculation shows that

$$
\frac{1+b^{2}}{1-b^{2}}=\frac{1}{\sqrt{1-r^{2}}} ; \frac{1}{1+a^{2}}=\frac{1+\sqrt{1-s^{2}}}{2} .
$$


Then

$$
I_{2}=\frac{1}{\sqrt{1-r^{2}}}\left\{\log \left(\frac{1+\sqrt{1-s^{2}}}{2}\right)+2 \log \left(1-\frac{\left[1-\sqrt{1-s^{2}}\right]\left[1-\sqrt{1-r^{2}}\right]}{s r}\right)\right\} .
$$

\section{Step 3: Combine the integrals}

From (3.14), (3.18), and the last identity,

$$
\begin{aligned}
I= & \frac{1}{\sqrt{\alpha \beta}}\left\{\log \left(1+\frac{\beta+\alpha}{2}\right)+\log \left(\frac{1+\sqrt{1-s^{2}}}{2}\right)\right. \\
& \left.+2 \ln \left(1-\frac{\left[1-\sqrt{1-s^{2}}\right]\left[1-\sqrt{1-r^{2}}\right]}{s r}\right)\right\} .
\end{aligned}
$$

Substituting for $s$ and $r$ gives (3.13).

We finish this section with evaluation of $G_{a}(c)$ for $a=2,3$ and $c=\frac{1}{8}, \frac{1}{4}$ respectively.

\section{Lemma 3.2}

$$
\begin{aligned}
& G_{2}\left(\frac{1}{8}\right)=0 \Leftrightarrow H_{2}(1)=\frac{1}{8} ; \\
& G_{3}\left(\frac{1}{4}\right)=0 \Leftrightarrow H_{3}(1)=\frac{1}{4} .
\end{aligned}
$$

\section{Proof}

Let us do (3.20). We have $a=2$ and $c=\frac{1}{8}$. By (2.6), $d=4 c=\frac{1}{2}$. So

$$
\frac{d+c}{d-c}=\frac{5}{3} ; \frac{d+c+2}{d-c}=7
$$

and hence

$$
\begin{aligned}
\frac{d+c}{d-c}-\sqrt{\left(\frac{d+c}{d-c}\right)^{2}-1} & =\frac{1}{3} \\
\frac{d+c+2}{d-c}-\sqrt{\left(\frac{d+c+2}{d-c}\right)^{2}-1} & =7-4 \sqrt{3} .
\end{aligned}
$$

Substitution in (2.10) and some manipulations show that

$$
\begin{aligned}
G_{2}\left(\frac{1}{8}\right) & =-4 \log 3-\log (7+4 \sqrt{3})+10 \log 2-4 \log \left(\frac{-4+4 \sqrt{3}}{3}\right) \\
& =2 \log 2-\log (7+4 \sqrt{3})-\log (\sqrt{3}-1)^{4} .
\end{aligned}
$$

A calculation shows that

$$
(\sqrt{3}-1)^{4}=2^{2}(7-4 \sqrt{3}) .
$$

Substitution then gives (3.20). The proof of (3.21) is similar. 


\section{The Proof of Theorem 2.3(A)}

We shall do this in five steps:

Step 1: Reduction to $P$ with zeros in $[0,1]$

Note first that if $a \in \mathbb{C}$, and $r \in[0,1]$,

$$
\frac{\max _{|z|=1}\left|\prod_{j=1}^{n}\left(z-a_{j}\right)\right|}{\min _{|z|=r}\left|\prod_{j=1}^{n}\left(z-a_{j}\right)\right|} \leq\left|\prod_{j=1}^{n} \frac{1+\left|a_{j}\right|}{r-\left|a_{j}\right|}\right| .
$$

Since we are looking for a lower bound for the linear Lebesgue measure of the set in $(1.1)$, it follows that it suffices to find a lower bound for $m_{1}(\mathcal{S})$, where

$$
\mathcal{S}:=\left\{r \in[0,1]: r^{a n}\left|\prod_{j=1}^{n}\left(\frac{1+\alpha_{j}}{r-\alpha_{j}}\right)\right|<\varepsilon^{n}\right\},
$$

and where all $\alpha_{j} \geq 0$. Next, note that we have also assumed that we have a polynomial of exact degree $n$. This may be achieved by adding some $\alpha_{j}=1$, which again reduces the size of $\mathcal{S}$. Finally, we note that we may assume that all $\alpha_{j} \leq 1$ : again, replacing any $\alpha_{j}>1$ by 1 increases $\left|\frac{1+\alpha_{j}}{r-\alpha_{j}}\right|$ and so reduces the size of $\mathcal{S}$. So, in the sequel, we assume that all $\alpha_{j} \in(0,1]$.

Let

$$
\mathcal{E}:=\left\{r \in[0,1]: r^{a n}\left|\prod_{j=1}^{n}\left(\frac{1+\alpha_{j}}{r-\alpha_{j}}\right)\right| \geq \varepsilon^{n}\right\},
$$

so that

$$
m_{1}(\mathcal{S})=1-m_{1}(\mathcal{E}) .
$$

We must look for an upper bound for $m_{1}(\mathcal{E})$.

Step 2: Show that $m_{1}(\mathcal{E})$ may be maximized by $\mathcal{E}$ of the form $[c, 1]$ Let

$$
\ell:=\sup m_{1}(\mathcal{E})
$$

where the sup is taken over all $\alpha_{1}, \alpha_{2}, \ldots \alpha_{n} \in[0,1]$ and the corresponding sets $\mathcal{E}$. We can extract a subsequence of $n$-tuples $\left(\alpha_{1}, \alpha_{2}, \ldots \alpha_{n}\right)$ converging to an $n$-tuple for which the sup is actually attained. (This is easy to see from (4.1) and regularity properties of $\left.m_{1}\right)$. Let us assume that $\left(\alpha_{1}, \alpha_{2}, \ldots \alpha_{n}\right)$ is such an $n$-tuple, and $\mathcal{E}$ is the corresponding set.

We first claim that $\mathcal{E} \subset[0,1]$ consists of finitely many intervals, some of which may degenerate to a single point. Moreover, each $\alpha_{j}$ lies in one of the intervals that has non-empty interior. To see this, note that

$$
f(r):=r^{-2 a n} \prod_{j=1}^{n}\left(\frac{r-\alpha_{j}}{1+\alpha_{j}}\right)^{2}-\varepsilon^{-2 n}
$$

is an analytic function of $r \in(0, \infty)$, (with the usual branch of $r^{-2 a n}$ ), so is either identically zero, or has finitely many zeros in every compact subinterval of $(0,1]$. Since each interval of $\mathcal{E}$ has zeros of $f$ (or the point 1) as endpoints, while if $a>0$, $\mathcal{E}$ clearly omits a neighbourhood of 0 , the claim about the finitely many intervals follows. (If $a=0, \mathcal{E}$ is a lemniscate of at most $n$ intervals). That each $\alpha_{j}$ lies in 
such an interval that is also non-empty follows directly from the definition of $\mathcal{E}$. We may omit the discrete points from $\mathcal{E}$ and assume that $\mathcal{E}$ is a union of, say, $k$ disjoint intervals

$$
\mathcal{E}=\bigcup_{j=1}^{k} I_{j}
$$

where

$$
I_{j}=\left[a_{j}, b_{j}\right] \text { and each } b_{j}<a_{j+1} .
$$

The linear measure of $\mathcal{E}$ is

$$
\ell=\sum_{j=1}^{k}\left(b_{j}-a_{j}\right)
$$

Define a strictly increasing piecewise linear map $h$ from $\mathcal{E}$ onto $[1-\ell, 1]$ that shifts each $I_{j}$ to the right until we have a single interval with 1 as right endpoint. The formula for $h$ is

$$
h(x):=x+1-\ell-a_{j}+\sum_{i=1}^{j-1}\left(b_{i}-a_{i}\right)=: x+A_{j}, x \in\left[a_{j}, b_{j}\right],
$$

$1 \leq j \leq k$. (The empty sum is interpreted as 0 ). Define

$$
\mathcal{E}_{1}:=\left\{s \in[0,1]: s^{a n}\left|\prod_{j=1}^{n} \frac{1+h\left(\alpha_{j}\right)}{s-h\left(\alpha_{j}\right)}\right| \geq \varepsilon^{n} .\right\}
$$

(Since each $\alpha_{j}$ lies in $\mathcal{E}$, each $h\left(\alpha_{j}\right)$ defined). We claim that

$$
[1-\ell, 1]=\mathcal{E}_{1},
$$

so that

$$
m_{1}\left(\mathcal{E}_{1}\right)=\ell=m_{1}(\mathcal{E})
$$

and then the proof of this step is complete. First note that as $h$ shifts intervals of $\mathcal{E}$ successively to the right,

$$
h(x) \geq x \geq 0, x \in \mathcal{E} .
$$

Moreover, $h$ preserves distances between points within each $I_{j}$, and reduces the distance between points in different intervals of $\mathcal{E}$, so

$$
|h(s)-h(t)| \leq|s-t|, s, t \in \mathcal{E} .
$$

Next, given $s \in[1-\ell, 1]$, we can write $s=h(r)$ for some $r \in \mathcal{E}$, and then

$$
\begin{aligned}
s^{a n} \prod_{j=1}^{n}\left|\frac{1+h\left(\alpha_{j}\right)}{s-h\left(\alpha_{j}\right)}\right| & =h(r)^{a n} \prod_{j=1}^{n}\left|\frac{1+h\left(\alpha_{j}\right)}{h(r)-h\left(\alpha_{j}\right)}\right| \\
& \geq r^{a n} \prod_{j=1}^{n}\left|\frac{1+\alpha_{j}}{r-\alpha_{j}}\right| \geq \varepsilon^{n},
\end{aligned}
$$

and then $[1-\ell, 1] \subseteq \mathcal{E}_{1}$. Since $\ell$ is maximal, we must have (4.3). So in the sequel, we assume that for some $c \geq 0$,

$$
\mathcal{E}=\left\{r \in[0,1]: r^{a n}\left|\prod_{j=1}^{n}\left(\frac{1+\alpha_{j}}{r-\alpha_{j}}\right)\right| \geq \varepsilon^{n}\right\}=[c, 1] .
$$

\section{Step 2: The basic inequality for $\mathcal{E}$}


Suppose first that $a>0$, so that in (4.4), $c>0$. Let $\nu$ be a unit measure with mass $\frac{1}{n}$ at each $\alpha_{j}$. Then we see with the notation

$$
U^{\nu}(z):=\int \log \frac{1}{|z-t|} d \nu(t)
$$

that

$$
[c, 1]=\mathcal{E}=\left\{r \in[0,1]: Q_{a}(r)+U^{\nu}(r)-U^{\nu}(-1) \geq \log \varepsilon\right\} .
$$

Now let $[c, d]=S\left(\mu_{a, c}\right)$, with the notation of the previous section. Then we have

$$
Q_{a}(r)+U^{\mu_{a, c}}(r)=F_{a, c} \text { in }[c, d] \subseteq[c, 1]
$$

and (4.5) gives

$$
U^{\mu_{a, c}}(r) \leq U^{\nu}(r)+\left[F_{a, c}-U^{\nu}(-1)-\log \varepsilon\right] \text { in }[c, d] .
$$

By the principle of domination [17, Thm. II.3.2, p.104], this last inequality holds for all $r \in \mathbb{C}$. In particular, choosing $r=-1$, we obtain

$$
U^{\mu_{a, c}}(-1) \leq F_{a, c}-\log \varepsilon
$$

and hence, with the notation (2.9),

$$
G_{a}(c) \leq-\log \varepsilon .
$$

If $-\log \varepsilon$ is in the range of $G_{a}$, we then obtain from (2.11),

$$
G_{a}(c) \leq G_{a}\left(H_{a}(\varepsilon)\right)
$$

and hence, as $G_{a}$ is decreasing,

$$
c \geq H_{a}(\varepsilon) .
$$

Then

$$
\begin{aligned}
& m_{1}(\mathcal{E})=1-c \leq 1-H_{a}(\varepsilon) \\
\Rightarrow & m_{1}(\mathcal{S})=1-m_{1}(\mathcal{E}) \geq H_{a}(\varepsilon),
\end{aligned}
$$

by (4.2). So we have completed the proof of Theorem 2.3(a) for $a>0$. Finally, for $a=0, Q_{a} \equiv 0$, and the above argument goes through even if $c=0$. Note that if $a=0$, the equilibrium measure $\mu_{a, c}$ is defined even for $c=0$, and is the classical equilibrium measure for $[0,1]$.

\section{The Proof of Theorem 2.3(B)}

\section{The Proof of Theorem 2.3(b)}

We shall use a crude discretisation procedure, of the type used in the theory of orthogonal polynomials in the 1980's. The finer method of Totik [17], [23] would yield sharper estimates, but those are not needed here. Fix $\varepsilon>0$ in the domain of $H_{a}$, let $\delta>0$, and choose $\varepsilon_{1}>\varepsilon$ such that

$$
H_{a}\left(\varepsilon_{1}\right)<H_{a}(\varepsilon)+\frac{\delta}{4}
$$

Let

$$
c:=H_{a}\left(\varepsilon_{1}\right) \Leftrightarrow G_{a}(c)=-\log \varepsilon_{1}
$$

and let, as in Section 2,

$$
S\left(\mu_{a, c}\right)=[c, d] .
$$


We emphasise that $c, d, \delta$ are fixed so do not change with $n$ below. Let $n \geq 1$ and choose

$$
c=t_{0}<t_{1}<t_{2}<\ldots<t_{n}=d
$$

such that if $J_{j}:=\left[t_{j}, t_{j+1}\right)$, then

$$
\int_{J_{j}} d \mu_{a, c}=\frac{1}{n}, 0 \leq j \leq n-1 .
$$

We set

$$
\left|J_{j}\right|:=t_{j+1}-t_{j}
$$

and assume that $\delta<(d-c) / 16$. (This is permissible, as $d, c$ approach the corresponding values for $H_{a}(\varepsilon)$ as $\left.\varepsilon_{1} \rightarrow \varepsilon+\right)$. It is easily seen from the explicit formula (2.7) for $\mu_{a, c}^{\prime}$ that it is bounded above and below by positive constants in $\left[c+\frac{\delta}{8}, d-\frac{\delta}{8}\right]$, and hence

$$
\frac{C_{1}}{n} \leq\left|J_{j}\right| \leq \frac{C_{2}}{n} \text { if } J_{j} \subset\left[c+\frac{\delta}{8}, d-\frac{\delta}{8}\right] .
$$

Here $C_{1}$ and $C_{2}$ are independent of $j, n$ (but depend on $\delta, a, c$ ). Moreover, the formula (2.7) implies that for some $C_{3}, C_{4}>0$

$$
n^{-C_{4}} \leq\left|J_{j}\right| \leq n^{-C_{3}}, 0 \leq j \leq n-1 .
$$

Finally, we can deduce from (2.7) that since $\mu_{a, c}^{\prime}$ does not change much in small intervals,

$$
C_{5} \leq\left|J_{j}\right| /\left|J_{j+1}\right| \leq C_{6}, 0 \leq j \leq n-2 .
$$

Again, $C_{i}, i=3,4,5,6$ are independent of $j, n$. Now consider

$$
\begin{aligned}
& \frac{1}{n} \log \left|r^{a n} \prod_{j=1}^{n}\left(\frac{1+t_{j}}{r-t_{j}}\right)\right|-\left\{Q_{a}(r)+U^{\mu_{a, c}}(r)-U^{\mu_{a, c}}(-1)\right\} \\
= & \sum_{j=0}^{n-1}\left\{\int_{J_{j}} \log \left(\frac{1+t_{j}}{1+t}\right) \mu_{a, c}^{\prime}(t) d t+\int_{J_{j}} \log \left|\frac{r-t}{r-t_{j}}\right| \mu_{a, c}^{\prime}(t) d t\right\} \\
= & : \sum_{j=0}^{n-1}\left(\Delta_{j, 1}+\Delta_{j, 2}\right) .
\end{aligned}
$$

Here for $t \in J_{j}$, uniformly in $t$ and $0 \leq j \leq n-1$,

$$
\log \left(\frac{1+t_{j}}{1+t}\right)=\log \left(1+\frac{t_{j}-t}{1+t}\right)=O\left(\left|J_{j}\right|\right)
$$

so as $\mu_{a, c}$ is a unit measure, (5.5) gives

$$
\sum_{j=0}^{n-1} \Delta_{j, 1}=O\left(n^{-C_{3}}\right) .
$$

Note that this sum is independent of $r$. Next, let

$$
r \in\left[c+\frac{\delta}{4}, d-\frac{\delta}{4}\right] \cup\left[d+\frac{\delta}{4}, 1\right] .
$$


If $r$ belongs to the first of these intervals, choose $j_{0}=j_{0}(r, n)$ such that $r \in J_{j_{0}}$. (If it belongs to the second interval, omit the estimation for $\left.J_{j_{0}}\right)$. Now if $\left|j-j_{0}\right| \geq 3$, we have for $t \in J_{j}$,

$$
\left|\frac{r-t}{r-t_{j}}-1\right|=\left|\frac{t-t_{j}}{r-t_{j}}\right| \leq \frac{\left|J_{j}\right|}{\left|r-t_{j}\right|} \leq \frac{\left|J_{j}\right|}{\left|J_{j}\right|+\left|J_{j \pm 1}\right|} \leq C_{0}<1
$$

where $C_{0}$ is independent of $r, j, n$. (If $r \notin[c, d]$, this holds for all $j$ and $n \geq n_{0}(\delta)$ ). We have used (5.6) here. Then

$$
|\log | \frac{r-t}{r-t_{j}}|| \leq C \frac{\left|J_{j}\right|}{|r-t|}, t \in J_{j}
$$

with $C$ independent of $n, j, r$ and

$$
\begin{aligned}
& \sum_{j:\left|j-j_{0}\right| \geq 3}\left|\Delta_{j, 2}\right| \\
\leq & \sum_{j:\left|j-j_{0}\right| \geq 3} \int_{J_{j}}|\log | \frac{r-t}{r-t_{j}}|| \mu_{a, c}^{\prime}(t) d t \\
\leq & C \max _{j}\left|J_{j}\right| \sum_{j:\left|j-j_{0}\right| \geq 3} \int_{J_{j}} \frac{1}{|r-t|} \mu_{a, c}^{\prime}(t) d t \\
\leq & C n^{-C_{3}} \log n=o(1) .
\end{aligned}
$$

Here we have used (5.5) and also the fact that we are restricting $r$ by (5.7) and the fact that $\mu_{a, c}^{\prime}$ is bounded in $\left[c+\frac{\delta}{8}, d-\frac{\delta}{8}\right]$. Finally, for $\left|j-j_{0}\right| \leq 2$, which occurs if $r$ belongs to the first interval in (5.7), we have

$$
\begin{aligned}
\Delta_{j, 2} & =\int_{J_{j}} \log \left|\frac{r-t}{r-t_{j}}\right| \mu_{a, c}^{\prime}(t) d t \\
& \geq C \int_{J_{j}} \log |r-t| d t \geq-C \frac{\log n}{n},
\end{aligned}
$$

by (5.4). Putting all these estimates together gives for $n \geq n_{0}(\delta)$ and uniformly for $r$ in the range (5.7),

$$
\begin{aligned}
& \frac{1}{n} \log \left|r^{a n} \prod_{j=1}^{n}\left(\frac{1+t_{j}}{r-t_{j}}\right)\right| \\
\geq & Q_{a}(r)+U^{\mu_{a, c}}(r)-U^{\mu_{a, c}}(-1)+o(1) \\
\geq & F_{a, c}-U^{\mu_{a, c}}(-1)+o(1) .
\end{aligned}
$$

Here we have used (2.4), which (as we noted) holds throughout $[c, 1]$. Now using our definition of $G_{a}$, and then (5.2), we continue this as

$$
\begin{aligned}
& =-G_{a}(c)+o(1) \\
& =\log \varepsilon_{1}+o(1) .
\end{aligned}
$$

The $o$ (1) term is uniform for $r$ in the range (5.7). Since $\varepsilon_{1}>\varepsilon$, it follows that for $n \geq n_{0}$, and for $r$ in the range (5.7),

$$
\left|r^{a n} \prod_{j=1}^{n}\left(\frac{1+t_{j}}{r-t_{j}}\right)\right| \geq \varepsilon^{n} .
$$


Thus

and hence

$$
m_{1}\left\{r \in[0,1]:\left|r^{a n} \prod_{j=1}^{n}\left(\frac{1+t_{j}}{r-t_{j}}\right)\right| \geq \varepsilon^{n}\right\} \geq 1-c-\frac{3}{4} \delta
$$

$$
m_{1}\left\{r \in[0,1]:\left|r^{a n} \prod_{j=1}^{n}\left(\frac{1+t_{j}}{r-t_{j}}\right)\right|<\varepsilon^{n}\right\} \leq c+\frac{3}{4} \delta<H_{a}(\varepsilon)+\delta,
$$

by (5.1) and (5.2). We have shown that for

$$
P(t):=\prod_{j=1}^{n}\left(t-t_{j}\right)
$$

the set in (1.1) has $m_{1}$ measure $<H_{a}(\varepsilon)+\delta$. Since $\delta>0$ may be made arbitrarily small (independently of $\varepsilon$ ), the proof of (b) is complete.

\section{The Proof of Theorems $2.4-2.6$}

\section{The Proof of Theorem 2.5}

Let $0<\tau<1$ and $\eta, \kappa>0$. Let $S$ be a monic polynomial, of degree $\ell$ say, such that $f S$ is analytic in $|z| \leq \tau$. We assume that $f$ itself is analytic on $|z|=\tau$. (If not, alter $\tau$ a little). We assume $n \geq \ell$ and write $[\lambda n / n]=p_{n} / q_{n}$ and use the well known error formula for Padé approximation,

$$
(f-[\lambda n / n])(z)=\frac{1}{2 \pi i} \int_{|t|=\tau} \frac{\left(f S q_{n}\right)(t)}{\left(S q_{n}\right)(z)}\left(\frac{z}{t}\right)^{(\lambda+1) n+1} \frac{d t}{t-z},|z|<\tau .
$$

This is a simple consequence of Cauchy' integral formula, see e.g. [1]. We deduce that for $r<\tau$,

$\max _{|z|=r} \frac{|f-[\lambda n / n]|(z)}{|z|^{\rho n}} \leq \frac{C \tau^{-\rho(n+\ell)} r^{-(\lambda+1-\rho) \ell}}{\tau-r}\left\{\left(\frac{r}{\tau}\right)^{(\lambda+1-\rho)(n+\ell)} \frac{\max _{|t|=\tau}\left|S q_{n}\right|(t)}{\min _{|z|=r}\left|S q_{n}\right|(z)}\right\}$

where $C$ depends only on $f, \ell, \tau, \lambda$. Defining

$$
P_{n+\ell}(z):=\left(S q_{n}\right)(\tau z) ; r^{\prime}:=r / \tau,
$$

we have

$$
\begin{gathered}
\max _{|z|=r} \frac{|f-[\lambda n / n]|(z)}{|z|^{\rho n} \leq} \frac{C \tau^{-\rho(n+\ell)} r^{-(\lambda+1-\rho) \ell}}{\tau-r}\left\{\left(r^{\prime}\right)^{(\lambda+1-\rho)(n+\ell)} \frac{\max _{|t|=1}\left|P_{n+\ell}\right|(t)}{\min _{|z|=r^{\prime}}\left|P_{n+\ell}\right|(z)}\right\} \\
\leq \frac{C \tau^{-\rho(n+\ell)} r^{-(\lambda+1-\rho) \ell}}{\tau-r} \eta^{n+\ell}
\end{gathered}
$$

for $r^{\prime} \in \mathcal{S}_{n}$, with

$$
m_{1}\left(\mathcal{S}_{n}\right) \geq H_{\lambda+1-\rho}(\eta),
$$

by Theorem 2.3. If we fix $\varepsilon$ and choose $\tau, \eta$ such that

$$
\tau^{-\rho} \eta<\varepsilon
$$

we obtain for $n \geq n_{0}$ (which depends on $C, \tau, \rho, \lambda, \kappa, \varepsilon, \ell$ ),

$$
\max _{|z|=r} \frac{|f-[\lambda n / n]|(z)}{|z|^{\rho n}} \leq \varepsilon^{n}
$$

such that

$$
r^{\prime} \in \mathcal{S}_{n} ; r \notin[0, \kappa] \cup[\tau-\kappa, \tau] .
$$


The measure of $r \in[0,1]$ for which (6.2) holds for $n \geq n_{0}$ is then at least

$$
\tau m_{1}\left(\mathcal{S}_{n}\right)-2 \kappa \geq \tau H_{\lambda+1-\rho}(\eta)-2 \kappa \text {. }
$$

By choosing $\kappa$ small enough, $\tau$ close enough to 1 , and $\eta$ close enough to $\varepsilon$, while satisfying (6.1), we may ensure that the measure of such $r$ exceeds $H_{\lambda+1-\rho}(\varepsilon)-\delta$, for a given $\delta>0$.

\section{Proof of Theorem 2.4}

This follows from the case $\rho=0, \varepsilon$ close to 1 , of Theorem 2.5.

\section{Proof of Theorem 2.6}

It is well known that for $n \geq \ell$, there exists a rational function $p_{n}^{*} / q_{n}^{*}$, with $p_{n}^{*}, q_{n}^{*}$ of degree $\leq n$, such that

$$
\left\|f-\frac{p_{n}^{*}}{q_{n}^{*}}\right\|_{L_{\infty}(|z|=1)}=\rho_{n}(f) .
$$

Write $[n / n]=p_{n} / q_{n}$ and let $S$ be the polynomial of degree $\ell$ such that $f S$ is analytic in $\{z:|z| \leq 1\}$. Then the contour integral error formula gives for $n \geq \ell$ and $|z|<1$,

$$
\begin{aligned}
\frac{\left(f q_{n}-p_{n}\right)(z)\left(S q_{n-\ell}^{*}\right)(z)}{z^{2 n+1}} & =\frac{1}{2 \pi i} \int_{|t|=1} \frac{\left(f q_{n} S q_{n-\ell}^{*}\right)(t)}{t^{2 n+1}(t-z)} d t \\
& =\frac{1}{2 \pi i} \int_{|t|=1} \frac{\left.\left(f q_{n-\ell}^{*}-p_{n-\ell}^{*}\right)(t) S q_{n}\right)(t)}{t^{2 n+1}(t-z)} d t .
\end{aligned}
$$

From this we derive the estimate

$$
\max _{|z|=r}|f-[n / n]|(z) \leq \frac{C}{1-r} \rho_{n-\ell}(f)\left\{r^{2 n} \frac{\max _{|t|=1}\left|q_{n-\ell}^{*} S q_{n}\right|(t)}{\min _{|z|=r}\left|q_{n-\ell}^{*} S q_{n}\right|(z)}\right\},
$$

where $C$ is independent of $n, r$. Now we apply Theorem 2.3 with $a=1$ (for $q_{n-\ell}^{*} S q_{n}$ has degree $\leq 2 n)$ to deduce the result, as above.

\section{REFERENCES}

[1] G.A. Baker and P.R. Graves-Morris, Padé Approximants, 2nd Edn., Encyclopaedia of Mathematics and its Applications, Vol. 59, Cambridge University Press, Cambridge, 1996.

[2] P. Borwein, T. Erdelyi, Polynomials and Polynomial Inequalities, Springer, New York, 1995.

[3] I.S. Gradshteyn, I.M. Ryzhik, Table of Integrals, Series and Products, Academic Press, San Diego, 1980.

[4] A.L. Levin and E.B. Saff, Fast Decreasing Rational Functions, Israel Journal of Maths., 114(1999), 125-148.

[5] G.G. Lorentz, M. von Golitschek, Y. Makovoz, Constructive Approximation: Advanced Problems, Grundlehren der Mathematischen Wissenschaften, Vol. 304, Springer, Berlin, 1996.

[6] D.S. Lubinsky, Diagonal Padé Approximants and Capacity, J. Math. Anal. Applns., 78(1980), $58-67$.

[7] D.S. Lubinsky, Divergence of Complex Rational Approximations, Pacific Journal of Mathematics, 108(1983), 141-153.

[8] D.S. Lubinsky, On the Diagonal Padé Approximants of Meromorphic Functions, Indagationes Mathematicae, 7(1996), 97-110.

[9] D.S. Lubinsky, Will Ramanujan kill Baker-Gammel-Wills? (A Selective Survey of Padé Approximation), (in) Proceedings of IDOMAT98, International Series in Numerical Mathematics, Vol. 132, Birkhauser, Basel, 1999, pp. 159-174.

[10] D.S. Lubinsky, On the Maximum and Minimum Modulus of Rational Functions, Canad. J. Math., 52(2000), 815-832. 
[11] D.S. Lubinsky, Sharp Estimates for the Maximum over Minimum Modulus of Rational Functions, to appear in Proceedings of the Amer. Math. Soc.

[12] H.N. Mhaskar, Introduction to the Theory of Weighted Polynomial Approximation, World Scientific, 1996

[13] J. Nuttall, Convergence of Padé Approximants of Meromorphic Functions, J. Math. Anal. Applns., 31(1970), 147-153.

[14] C. Pommerenke, Padé Approximants and Convergence in Capacity, J. Math. Anal. Applns., 41(1973), 775-780.

[15] E.A. Rakhmanov, On the Convergence of Padé Approximants in Classes of Holomorphic Functions, Math. USSR. Sbornik, 40(1981), 149-155.

[16] E.B. Saff, Incomplete and Orthogonal Polynomials, (in) Approximation Theory IV, (eds. C.K. Chui, L.L. Schumaker, J.D. Ward), Academic Press, Orlando, 1983, pp. 219-256.

[17] E.B. Saff and V. Totik, Logarithmic Potential with External Fields, Springer, Berlin, 1997.

[18] E.B. Saff, J.L. Ullman, R.S. Varga, Incomplete Polynomials: an Electrostatics Approach, (in) Approximation Theory III (ed. E.W. Cheney), Academic Press, San Diego, 1980, pp. 769-782.

[19] H. Stahl, Beiträge zum Problem der Konvergenz von Padé Approximierenden, Dissertation, Technische Universität Berlin, 1976.

[20] H. Stahl, General Convergence Results for Padé Approximants, (in) Approximation Theory VI, (eds. C.K. Chui, L.L. Schumaker, J.D. Ward), Academic Press, San Diego, 1989, pp.605634.

[21] H. Stahl, The Convergence of Padé Approximants to Functions with Branch Points, J. Approx. Theory, 91(1997), 139-204.

[22] H. Stahl, Conjectures Around the Baker-Gammel-Wills Conjecture: Research Problems 97-2, Constr. Approx., 13(1997), 287-292.

[23] V. Totik, Weighted Approximation with Varying Weights, Springer Lecture Notes in Mathematics, Vol. 1569, Springer, Berlin, 1994.

The John Knopfmacher Centre for Applicable Analysis and Number Theory, Department of Mathematics, Witwatersrand University, Wits 2050, South Africa.

E-mail address: 036dsl@cosmos.wits.ac.za 